\title{
Analysis of Radiotherapy Impact on Survival in Resected Stage I/ii Pancreatic Cancer Patients: A Population-based Study
}

\section{Dong Han}

Beijing Hospital of Traditional Chinese Medicine

\section{Fei Gao}

Beijing Hospital of Traditional Chinese Medicine

JinLong Liu

LuanPing Hospital of Traditional Chinese Medicine

\section{Hao Wang}

Beijing Hospital of Traditional Chinese

Qi Fu ( $\nabla$ fuqizyyy@163.com )

Department of Oncology \& Hematology, Beijing Hospital of Traditional Chinese Medicine, Capital Medical University, Beijing, China;

\section{Research}

Keywords: Radiotherapy, Pancreatic cancer, SEER database, Survival analysis, Overall mortality

Posted Date: December 16th, 2020

DOI: https://doi.org/10.21203/rs.3.rs-126795/v1

License: @ (i) This work is licensed under a Creative Commons Attribution 4.0 International License. Read Full License 


\section{Abstract}

Background: The application of radiotherapy (RT) in pancreatic cancer remains controversial. The aim of the study was to evaluate the efficacy of radiotherapy (neoadjuvant and adjuvant radiotherapy) for resectable I/II pancreatic cancer.

Methods: 14977 patients with pancreatic cancer were identified from SEER database from 2004 to 2015. Multivariate analyses were performed to determine factors including RT on overall survival. Overall survival and overall mortality among the different groups were evaluated using the Kaplan-Meier method and Gray's test.

Results: Patients were divided into groups according to whether they received radiotherapy or not. The median survival time of all 14977 patients without RT was 20 months, neoadjuvant RT was 24 months and adjuvant RT was 23 months $(p<0.0001)$. Median survival time of 2089 stage I patients without RT was 56 months, significantly longer than those with RT regardless of neoadjuvant or adjuvant RT (no RT: 56 months vs adjuvant RT: 37 months vs neoadjuvant RT: 27 months, $P=0.0039$ ). Median survival time of 12888 stage II patients with neoadjuvant RT was 24 months, adjuvant RT 22 months, significantly prolonged than those without radiotherapy(neoadjuvant RT: 24 months vs adjuvant RT: 22 months vs no RT: 17 months, Pख0.0001). Neoadjuvant RT ( $\mathrm{HR}=1.434, P=0.023,95 \% \mathrm{Cl}$ : 1.051-1.957) was independent risk factors for prognosis of stage I patients, and adjuvant $\mathrm{RT}$ ( $\mathrm{HR}=0.904, \mathrm{P}<0.001,95 \% \mathrm{Cl}$ : 0.861-0.950) predicted better outcomes for prognosis of stage II patients by multivariate analysis. The risk of cancer-related death caused by neoadjuvant RT in stage I and no-RT in stage II patients were significantly higher.

Conclusions: The study identified a significant survival advantage for the use of adjuvant RT over surgery alone or neoadjuvant RT in treating stage II pancreatic cancer. RT was not associated with survival benifit in stage I patients.

\section{Background}

Pancreatic cancer (PC) is an extremely malignant tumor with poor outcomes. The 5 -year survival is as low as $2-9 \%$ [1-3]. The incidence rate of pancreatic cancer is increasing year by year [4], and it has been estimated to rise from the fourth cause of cancer-related deaths to the second major cause of cancer-related death in the United States [5].

Surgery remains the only curative treatment for PC. However, patients with PC usually present late period and only $20 \%$ of them have a chance of undergoing surgery when diagnosed [6]. Even in resectable patients who received surgery treatment, the prognosis was not very satisfactory. Adjuvant treatment is recommended in resected pancreatic cancer with PT1-4/N0-1M0 who undergo an R0/R1 resection to reduce the recurrence rate. In the past decades, owing to the chemotherapy and radiotherapy(RT) technology development, for patients who can successfully receive surgical resection, the 5-year survival rate after adjuvant treatment accounts for $27 \%$ [7].

Chemoradiation technology has been used for resectable PC. In theory, the goal of preoperative chemoradiotherapy for PC is to purify vascular boundary, increase the possibility of negative resection at the margin and prolong survival of early treatment of micrometastatic disease. Postoperative radiotherapy can provide sufficient local control to prevent or delay the local lesions progression, and postoperative chemotherapy can reduce the recurrence rate. However, in fact, the effect of radiotherapy on the prognosis of resectable PC is controversial.

Results from ESPAC-1 trial revealed that postoperative chemoradiotherapy may not be necessary or even harmful [8]. But the conclusion is controversial due to the lack of quality control of radiation treatment. In an open-label, multicenter, randomized phase III trial [9], 132 resected patients with R0/R1 received either chemoradiotherapy group or chemotherapy group, median survival time were 26.5 and 28.5 months, respectively $(P>0.05)$. The results indicated chemoradiotherapy did not improve the survival compared with chemotherapy. A meta-analysis [10] included five randomised controlled trials of adjuvant treatment in pancreatic adenocarcinoma 939 patients, and the results showed chemoradiation is not effective adjuvant treatment in pancreatic cancer. 
In contrast, other studies obtained different conclusions about the effect of radiotherapy. In the GERCOR phase II study[11], 90 patients after $\mathrm{RO}$ resection of pancreatic head cancer were randomly divided into four gemcitabine treatment cycles or two gemcitabine cycles followed by gemcitabine with concurrent radiation weekly(50.4 Gy/28f), the results showed median disease-free survival(DFS)was 12 months in the adjuvant chemoradiotherapy group and 11 months in the chemotherapy alone group. First local recurrence was notably lower in the chemoradiotherapy group (11\% v $24 \%$ ).

The contradiction of the conclusions from different studies leads to the lack of clear guidelines for the application of radiotherapy in pancreatic cancer. This retrospective study was based on a large-scale population database to evaluate the efficacy of radiotherapy (neoadjuvant and adjuvant radiotherapy) for resectable I/II PC.

\section{Materials And Methods}

SEER (Surveillance, Epidemiology, and End Results) database of the National Cancer Institute is an important resource for population-based oncology research, documenting the information of cancer patients in some states in the United States for 40 years, with information on the diagnosis, treatment and survival data of millions of confirmed cancer patients. The number of SEER registration stations has now been expanded to eighteen.

The study extracted data using SEER*stat 8.3.6 software. Permission to access the custom data file in the SEER program was obtained and the reference number was 10016-Nov2019. All study variables were obtained directly from the SEER database. This study was approved by Ethics Committee of Beijing Hospital of Traditional Chinese Medicine, Capital Medical University.

We extracted pancreatic cancer patients' records data registered in SEER database from 2004 to 2015 and patients who accord with the following criteria were included in the study: (1) Adults aged $\geq 18$; (2) Pathologically confirmed malignant tumor originated from pancreatic duct epithelium; (3) Patients who diagnosed as stage I/II pancreatic cancer according to the American Joint Committe on cancer (AJCC)staging manual (Stage I-T1: localized within the pancreas, with maximum diameter $\leq 2 \mathrm{~cm}$ or T2: localized within the pancreas, with a maximum diameter $>2 \mathrm{~cm}$; N0: no regional lymph node metastasis; M0: no distant metastasis. Stage II-T3: extends beyond the pancreas but not involving the celiac axis or superior mesenteric artery; N0 and M0 or T1-T3/N1: regional lymph node metastasis and M0); (4) patients who received surgical resection; (5) Complete radiotherapy information record (including neoadjuvant RT, adjuvant RT and non-RT). (6) Survival time $\geq 1$ month. Figure 1 displayed the cohort identification process.

The primary outcome of the study was overall survival. Overall survival was determined from the beginning of the diagnosis until death of any cause or until the last follow-up date. Secondary endpoints were cancer-related mortality. Variables extracted from the SEER database included the following: age at diagnosis, year of diagnosis, sex, race recode, primary site, ICD-0-3 Hist/behave, pathological grade, derived AJCC T, RX Summ-Surg Prim Site, radiation sequence with surgery, chemotherapy recode, survival months, vital status recode, cause of death (COD) to site recode, Cause-specific death classification, Other cause of death classification.

All statistical calculations were carried out using SPSS 19.0 software, R(version 3.6.3) and figures were drawn by Graphpad Prism 7.0 and R. All variables have been converted to category variables for better analysis. The clinical characteristics baseline among different groups were compared by Chi-square test. The overall survival analysis was estimated by Kaplan-Meier curves and compared by the Log-Rank method. The Hazard's ratio was determined by univariate and multivariate Cox proportional hazard model. Secondary endpoint was compared using competitive risk analysis. Competition risk analysis was conducted by Gray's test using "cmprsk" and "survival" packages in R. All statistical tests were two-sided, and P-value $<0.05$ was considered statistically significant.

\section{Results}

The correlation between clinical parameters and RT in pancreatic cancer patients 
From the year 2004 until the end of the year 2015, 164035 patients with pancreatic cancer were enrolled in SEER database. In total, there were 2089 stage I patients and 12888 stage II patients underwent surgery operation were enrolled in this study. The median age at diagnosis was 67 years old (age ranging from 18 to 97). Among stage I patients, 88 patients received neoadjuvant RT and 388 patients received adjuvant RT. The proportion of patients receiving radiotherapy differed in year of diagnosis, sex, race, primary site, pathological grade, $T$ stage and whether received chemotherapy.

544 patients received neoadjuvant RT and 4200 patients received adjuvant RT in stage II patients. The composition ratio of radiation therapy in stage II patients differed in age, year of diagnosis, race, primary site, pathological grade, $\mathrm{T}$ stage, $\mathrm{N}$ stage, whether received chemotherapy and surgical methods $(P<0.05)$. The characteristics of all patients are presented in Table 1.

\section{Overall survival analysis of Stage I/II pancreatic cancer patients}

14977 patients with pancreatic cancer were divided into three groups according to whether they received RT or not. KaplanMeier (KM) analysis showed that the median survival time of patients without RT was 20 months, neoadjuvant RT was 24 months and adjuvant RT was 23 months $(p<0.0001$, Figure $2 A)$. We performed subgroup analysis according to different stages.

2089 cases of stage I pancreatic cancer patients were divided into three groups by radiotherapy. Comparison of the median survival time differences was conducted in the three groups. Median survival time of patients without RT was 56 months, significantly longer than the median survival time of those with RT regardless of neoadjuvant or adjuvant RT (no RT: 56 months vs adjuvant RT: 37 months vs neoadjuvant RT: 27 months, $P=0.0039$, Figure 2B). Similarly, all 12888 patients with stage II pancreatic cancer were divided into three groups according to whether they received radiotherapy or not. Median survival time of patients with neoadjuvant RT was 24 months, adjuvant RT 22 months, significantly prolonged than the median survival time of those without radiotherapy(neoadjuvant RT: 24 months vs adjuvant RT: 22 months vs no RT: 17 months, P凶0.0001, Figure 2C).

\section{Univariate analysis of clinical features affecting the prognosis}

Univariate survival analysis was separately performed for stage I/II patients. For stage I patients, the unadjusted protective factor was tumor located in tail of pancreas. Radiotherapy including age ( $\geq 60$ years old), male, pathology with worse differentiation, higher $\mathrm{T}$ stage were adverse diagnostic factors.

For patients with stage II pancreatic cancer, radiotherapy, chemotherapy and newly diagnosed pancreatic cancer after 2010 were the advantageous factors for the prognosis of patients. The harmful factors for the prognosis included age ( $\geq 60$ years old), male, pathology with worse differentiation, higher T and higher $\mathrm{N}$ stage. The results are shown in Table 2.

\section{Multivariate analysis of clinical features affecting the prognosis}

Multivariate survival analysis of stage I pancreatic cancer showed that neoadjuvant RT (HR=1.434, $P=0.023,95 \% \mathrm{Cl}$ : 1.051 1.957 ), age ( $\geq 60$ years old), male, pathology with worse differentiation, higher $T$ stage were independent risk factors for prognosis, indicating a shorter survival period. Chemotherapy, tumor located in body and tail of pancreas, non-white/black Americans were favorable prognostic factors and related to longer survival period.

Also, we conducted multivariate survival analysis for stage II pancreatic cancer patients. Results showed that adjuvant RT $(\mathrm{HR}=0.904, \mathrm{P}<0.001,95 \% \mathrm{Cl}: 0.861-0.950)$ including chemotherapy, expanded surgical procedure, pancreatic duct tumors, diagnosed pancreatic cancer after 2010 and non-black Americans predicted better outcomes. On the contrary, age ( $\geq 60$ years old), male, pathology with worse differentiation, higher $\mathrm{T}$ and $\mathrm{N}$ stage predicted worse outcomes, which means shorter survival time. Multivariate analysis results are shown in Table 3.

\section{Competitive risk analysis for cancer-related death of I/II pancreatic cancer patients}


The causes of death were divided into cancer-related death and non-cancer-related death. We analyzed the death outcomes by competitive risk model. The results showed that the 1-, 3-, and 5-year cancer-related mortality rate in stage I patients treated with neoadjuvant RT were $13.98 \%, 52.33 \%$ and $63.58 \%$ respectively. In adjuvant RT group, the 1-year, 3-year and 5-year cancerrelated mortality rates were $10.95 \%, 43.16 \%$ and $48.94 \%$, nevertheless these rates decreased to $11.54 \%, 32.02 \%$ and $39.51 \%$ respectively in patients without radiotherapy. The risk of cancer-related death caused by neoadjuvant RT was significantly higher than adjuvant RT and no-RT ( $p<0.001$; Figure $3 A)$.

The 1-year, 3-year and 5-year cancer-related mortality rates in patients with stage II pancreatic cancer without radiotherapy were $32.70 \%, 66.78 \%$ and $74.16 \%$ respectively, In patients who received adjuvant RT, the 1-year, 3-year and 5-year cancer-related mortality rates were $21.16 \%, 63.47 \%$ and $73.38 \%$. The rates were $17.57 \%, 55.30 \%$ and $68.49 \%$ in neoadjuvant RT group. The results showed the cancer-related death of II stage patients without radiotherapy was significantly higher than that of patients received radiotherapy $(P<0.001$; Figure $3 B)$.

\section{Discussion}

Although PC in early stage can be treated surgically, the complete resection rate is low due to anatomical position of pancreas complexity, the recurrence rate can up to $80 \%$ [12]. Previous studies have confirmed compared with surgery treatment only, postoperative adjuvant chemotherapy has positive effect by preventing or delaying tumor recurrence and improving the longterm survival rate. In CONKO-001 phase III clinical trial [13, 14], 354 patients with PC after resection were randomly divided into adjuvant gemcitabine (GEM) treatment group or observation group. The results showed GEM adjuvant chemotherapy improved Disease-free Survival(DFS) (13.4 months vs 6.9 months, $P<0.001)$. The long-term follow-up results showed that the total survival time of patients in GEM group was significantly superior than observation group (the mOS was 22.8 months vs 20.2 months, the 5 -year survival rate was $20.7 \%$ vs $10.4 \%$, and the 10 -year survival rate was $12.2 \%$ vs $7.7 \%$, respectively, $p<0.01$ ) .The study supported conclusion that adjuvant chemotherapy compared with observation alone resulted in increased overall survival. Our study also showed that chemotherapy is an independent protective factor for the prognosis of pancreatic cancer. Chemotherapy reduced the risk of death by $17.3 \%$ in patients with stage I and $37.2 \%$ in patients with stage II.

Adjuvant chemotherapy is necessary for resectable pancreatic cancer, but the role of radiotherapy remains controversial due to opposite conclusions drawn from different studies. The results of EROTC-40891 showed that there was no significant difference in survival rate and progression free survival rate between the chemoradiotherapy group and the observation group, and adjuvant chemoradiotherapy did not improve survival [7]. Results from ESPAC-1 trial identified adjuvant chemotherapy has significant survival benefits for patients with pancreatectomy, while adjuvant chemoradiotherapy has adverse effects on survival $[8,15,16]$. But other studies held the opposite conclusion. Since a randomized, prospective, multi-institutional Gastrointestinal Tumor Study Group (GITSG) trial $[17,18]$ had supported postoperative adjuvant radiotherapy and chemotherapy, subsequent clinical trials also demonstrated a fact chemoradiotherapy had survival advantage for resected patients. Results from a prospective database of 616 pancreatic cancer patients after resection of pancreatic cancer at Johns Hopkins Hospital suggested adjuvant chemoradiotherapy significantly improves survival when compared with patients observation alone [19]. The Mayo Clinic conducted a retrospective review of 466 patients who underwent R0 resection of pancreatic cancer, and found that overall survival was better in patients who received adjuvant chemoradiotherapy than those not received adjuvant chemoradiotherapy. Also, there were two large-scale population-based retrospective studies supported radiotherapy as an adjuvant treatment for localized pancreatic cancer. In 2008, Stessin et al.[20] analyzed 3885 cases of surgically resected stage I/II pancreatic cancer patients in SEER database from 1994 to 2003. Overall survival of patients receiving neoadjuvant RT was 23 months, while patients without RT was 12 months, and patients receiving adjuvant RT was 17 months. The analysis showed a survival benefit for the use of neoadjuvant RT in treating pancreatic cancer. In 2010, McDade et al. [21] analyzed 5676 cases of resected pancreatic adenocarcinoma in SEER database from 1988 to 2005. The median survival time (18 months) of patients with adjuvant radiotherapy was better than those without adjuvant radiotherapy (10 months, $\mathrm{P}<0.0001)$. These studies have come to different conclusions presumably because of the patients included with different baseline characteristics, staging, treatment methods and doses. 
Neoadjuvant radiotherapy alone was rarely used in patients with resectable pancreatic cancer. First, pancreatic cancer represents highly resistant to radiation, the response rate to radiotherapy was low. Second, the pancreas is a retroperitoneal organ with a deep anatomic position, surrounded by important organs such as stomach, liver and other organs. The effect of radiotherapy is affected by many factors such as target area delineation, peristalsis of surrounding organs. Another barrier is the intolerance of surrounding organs to high dose radiotherapy, which leads to the radiation effect limitation. Furthermore, the use of neoadjuvant radiotherapy for resectable pancreatic cancer may delay surgery, providing an opportunity for tumor metastasis that becomes unresectable. These barriers limited the application of neoadjuvant radiotherapy in pancreatic cancer. Our results displayed in the resectable pancreatic cancer, the number of patients receiving neoadjuvant radiotherapy was small, only $88(4.21 \%)$ in stage I and $544(4.22 \%)$ in stage II patients.

Different from the studies of Stessin and McDade, we performed a subgroup analysis of resectable pancreatic cancer according to TNM staging. Dramatic differences are observed in radiotherapy effect on stage I and II pancreatic cancer patients. Our study showed that for stage I pancreatic cancer patients, KM analysis indicated the median survival time of patients with no radiotherapy was significantly longer than those with radiotherapy, and the median survival time of patients received neoadjuvant radiotherapy is the shortest (56 months with no RT vs 37 months with adjuvant RT vs 27 months with neoadjuvant RT, $P=0.0039$, Fig. 2A). Multivariate Cox analysis showed that neoadjuvant radiotherapy was an independent risk factor. The 1-, 3-and 5-year risk of cancer-related death of neoadjuvant radiotherapy was significantly higher than nonradiotherapy and adjuvant radiotherapy. Similar to the previous study, Hazard et al.[22] analyzed 3008 pancreatic patients who underwent resection without distant metastasis from 1988 to 2002 in SEER database. Multivariate analysis showed that radiation therapy played a positive role in overall survival for patients who had direct extension beyond the pancreas and/or regional lymph node involvement $(P<0.01)$ but not for patients with T1-T2N0M0 disease $(P>0.05)$. It is noteworthy that in our study, Among stage I patients received neoadjuvant radiotherapy, of whom 15 cases underwent total pancreatectomy and subtotal gastrectomy or duodenectomy/extended pancreatoduodenectomy. We speculated these 15 patients may have highrisk factors that require neoadjuvant chemotherapy to improve R0 resection rate, the characteristics of these patients may mean a worse prognosis, leading to the conclusion that neoadjuvant radiotherapy has a negative effect in stage I patients.

For patients with stage II pancreatic cancer, the effect of radiotherapy on survival was completely opposite. KM analysis indicated that radiotherapy prolonged the patients' survival time, and the median survival of patients without radiotherapy is the shortest ( 17 months with no RT vs 22 months with adjuvant RT vs 24 months with neoadjuvant RT, $\mathrm{P}<0.0001, \mathrm{Fig}$. 2B). Cox analysis showed that adjuvant radiotherapy is an independent protective factor. Cancer-related death risk of no radiotherapy in 1-, 3-and 5-year was significantly higher than neoadjuvant and adjuvant radiotherapy. Our conclusion was consistent with Moody's study. Moody et al. [23] analyzed the record of 3252 patients with pancreatic cancer after operation in SEER database, the results showed that radiotherapy could significantly improve the survival time of patients with stage II B (T1-T3N1).

Because the sequence of radiotherapy and chemotherapy was not recorded in SEER database, we had to analyze radiotherapy and chemotherapy separately. Our results demonstrated that the addition of adjuvant radiotherapy could prolong the survival period and reduce tumor related death for stage II pancreatic cancer.

\section{Conclusion}

By the analysis of resectable stage I/II pancreatic cancer subgroups, our results demonstrated a survival benefit for adjuvant RT use in resected stage II pancreatic cancer. RT especially neoadjuvant RT may be associated with a worse survival in stage I patients. Our study was based on a large national cancer registration database to provide more evidence for the application of radiotherapy in operable stage $\mathrm{I} / \mathrm{Il}$ pancreatic cancer patients. It is undeniable that our research was a retrospective study and bias was inevitable. We try to minimize this bias through a large data analysis and statistical method. Also, other important prognostic factors, including surgical margins, lymphovascular invasion, performance status and comorbidities of patients, and chemotherapy information did not included in the study due to unavailable in SEER database, we did not incorporate these factors into our study, which led to some limitations. So further prospective clinical trials are needed.

\section{Abbreviations}


SEER

Surveillance, Epidemiology, and End Results; RT:Radiotherapy; OS:Overall survival; PC:Pancreatic cancer (PC); AJCC:American Joint Committe on cancer; COD:Cause of death; KM:Kaplan-Meier; DFS:Disease-free Survival; GEM:Gemcitabine; GITSG:Gastrointestinal Tumor Study Group.

\section{Declarations}

\section{Ethics approval and consent to participate}

Not applicable.

\section{Consent for publication}

Not applicable.

\section{Availability of data and materials}

The datasets analyzed in this study can be found in the SEER website of the National Cancer Institute (http://seer.cancer.gov).

\section{Competing interests}

The authors declare that they have no competing interests.

\section{Funding}

The research for this manuscript was not financially supported and none of the authors had any relevant financial relationships.

\section{Author Contributions}

DH: collected the data, analysis, and writing original manuscript. FG: provided statistical analysis and editing original manuscript. JL L: assisted in providing statistical analysis and editing original manuscript. HW: Data curation and editing original draft. Qi Fu: provided the original idea of the manuscript and reviewed the manuscript. All authors read and approved the final manuscript.

\section{Acknowledgments}

We would like to give special thanks to the efforts of the SEER tumor registry team.

\section{References}

1. Luo J, Xiao L, Wu C, Zheng Y, Zhao N. The incidence and survival rate of population-based pancreatic cancer patients: Shanghai Cancer Registry 2004-2009. PloS one. 2013;8(10):e76052.

2. McGuigan A, Kelly P, Turkington RC, Jones C, Coleman HG, McCain RS. Pancreatic cancer: A review of clinical diagnosis, epidemiology, treatment and outcomes. World journal of gastroenterology. 2018;24(43):4846-61.

3. Siegel RL, Miller KD, Jemal A. Cancer statistics, 2019. CA: a cancer journal for clinicians. 2019;69(1):7-34.

4. Saad AM, Turk T, Al-Husseini MJ, Abdel-Rahman O. Trends in pancreatic adenocarcinoma incidence and mortality in the United States in the last four decades; a SEER-based study2018;18(1):688.

5. Rahib L, Smith BD, Aizenberg R, Rosenzweig AB, Fleshman JM, Matrisian LM. Projecting cancer incidence and deaths to 2030: the unexpected burden of thyroid, liver, and pancreas cancers in the United States. Cancer research.

2014;74(11):2913-21.

6. Vincent A, Herman J, Schulick R, Hruban RH, Goggins M. Pancreatic cancer. The Lancet. 2011;378(9791):607-20. 
7. Smeenk HG, van Eijck CH, Hop WC, Erdmann J, Tran KC, Debois M, van Cutsem E, van Dekken H, Klinkenbijl JH, Jeekel J. Long-term survival and metastatic pattern of pancreatic and periampullary cancer after adjuvant chemoradiation or observation: long-term results of EORTC trial 40891. Annals of surgery. 2007;246(5):734-40.

8. Neoptolemos JP, Stocken DD, Friess H, Bassi C, Dunn JA, Hickey H, Beger H, Fernandez-Cruz L, Dervenis C, Lacaine F, Falconi M, Pederzoli P, Pap A, Spooner D, Kerr DJ, Büchler MW. A randomized trial of chemoradiotherapy and chemotherapy after resection of pancreatic cancer. The New England journal of medicine. 2004;350(12):1200-10.

9. Schmidt J, Abel U, Debus J, Harig S, Hoffmann K, Herrmann T, Bartsch D, Klein J, Mansmann U, Jäger D, Capussotti L, Kunz R, Büchler MW. Open-label, multicenter, randomized phase III trial of adjuvant chemoradiation plus interferon Alfa-2b versus fluorouracil and folinic acid for patients with resected pancreatic adenocarcinoma. Journal of clinical oncology : official journal of the American Society of Clinical Oncology. 2012;30(33):4077-83.

10. Stocken DD, Buchler MW, Dervenis C, Bassi C, Jeekel H, Klinkenbijl JH, Bakkevold KE, Takada T, Amano H, Neoptolemos JP, Pancreatic Cancer Meta-analysis G. Meta-analysis of randomised adjuvant therapy trials for pancreatic cancer. British journal of cancer. 2005;92(8):1372-81.

11. Van Laethem JL, Hammel P, Mornex F, Azria D, Van Tienhoven G, Vergauwe P, Peeters M, Polus M, Praet M, Mauer M, Collette L, Budach V, Lutz M, Van Cutsem E, Haustermans K. Adjuvant gemcitabine alone versus gemcitabine-based chemoradiotherapy after curative resection for pancreatic cancer: a randomized EORTC-40013-22012/FFCD9203/GERCOR phase II study. Journal of clinical oncology : official journal of the American Society of Clinical Oncology. 2010;28(29):4450-6.

12. Garrido-Laguna I, Hidalgo M. Pancreatic cancer: from state-of-the-art treatments to promising novel therapies. Nature reviews Clinical oncology. 2015;12(6):319-34.

13. Rieß H, Neuhaus P, Post S, Gellert K, Ridwelski K, Schramm H. CONKO-001: Final results of the randomized, prospective, multicenter phase III trial of adjuvant chemotherapy with gemcitabine versus observation in patients with resected pancreatic cancer (PC). Journal of clinical oncology : official journal of the American Society of Clinical Oncology. 2008;19:45-6.

14. Oettle H, Neuhaus P, Hochhaus A, Hartmann JT, Gellert K, Ridwelski K, Niedergethmann M, Zülke C, Fahlke J, Arning MB, Sinn $M$, Hinke A, Riess $H$. Adjuvant chemotherapy with gemcitabine and long-term outcomes among patients with resected pancreatic cancer: the CONKO-001 randomized trial. Jama. 2013;310(14):1473-81.

15. Neoptolemos JP, Dunn JA, Stocken DD, Almond J, Link K, Beger H, Bassi C, Falconi M, Pederzoli P, Dervenis C, FernandezCruz L, Lacaine F, Pap A, Spooner D, Kerr DJ, Friess H, Büchler MW. Adjuvant chemoradiotherapy and chemotherapy in resectable pancreatic cancer: a randomised controlled trial. Lancet (London, England). 2001;358(9293):1576-85.

16. Stocken DD, Büchler MW, Dervenis C, Bassi C, Jeekel H, Klinkenbijl JH, Bakkevold KE, Takada T, Amano H, Neoptolemos JP. Meta-analysis of randomised adjuvant therapy trials for pancreatic cancer. British journal of cancer. 2005;92(8):1372-81.

17. Kalser MH, Ellenberg SS. Pancreatic cancer. Adjuvant combined radiation and chemotherapy following curative resection. Archives of surgery (Chicago, III : 1960). 1985;120(8):899-903.

18. Further evidence of effective adjuvant combined radiation and chemotherapy following curative resection of pancreatic cancer. Gastrointestinal Tumor Study Group. Cancer. 1987;59(12):2006-10.

19. Herman JM, Swartz MJ, Hsu CC, Winter J, Pawlik TM, Sugar E, Robinson R, Laheru DA, Jaffee E, Hruban RH, Campbell KA, Wolfgang CL, Asrari F, Donehower R, Hidalgo M, Diaz LA, Jr., Yeo C, Cameron JL, Schulick RD, Abrams R. Analysis of fluorouracil-based adjuvant chemotherapy and radiation after pancreaticoduodenectomy for ductal adenocarcinoma of the pancreas: results of a large, prospectively collected database at the Johns Hopkins Hospital. Journal of clinical oncology : official journal of the American Society of Clinical Oncology. 2008;26(21):3503-10.

20. Stessin AM, Meyer JE, Sherr DL. Neoadjuvant radiation is associated with improved survival in patients with resectable pancreatic cancer: an analysis of data from the surveillance, epidemiology, and end results (SEER) registry. International journal of radiation oncology, biology, physics. 2008;72(4):1128-33.

21. McDade TP, Hill JS, Simons JP, Piperdi B, Ng SC, Zhou Z, Kadish SP, Fitzgerald TJ, Tseng JF. A national propensity-adjusted analysis of adjuvant radiotherapy in the treatment of resected pancreatic adenocarcinoma. Cancer. 2010;116(13):3257-66. 
22. Hazard L, Tward JD, Szabo A, Shrieve DC. Radiation therapy is associated with improved survival in patients with pancreatic adenocarcinoma: results of a study from the Surveillance, Epidemiology, and End Results (SEER) registry data. Cancer. 2007;110(10):2191-201.

23. Moody JS, Sawrie SM, Kozak KR, Plastaras JP, Howard G, Bonner JA. Adjuvant radiotherapy for pancreatic cancer is associated with a survival benefit primarily in stage IIB patients. Journal of gastroenterology. 2009;44(1):84-91.

\section{Tables}




\begin{tabular}{|c|c|c|c|c|c|c|c|c|}
\hline \multirow{2}{*}{$\begin{array}{l}\text { Clinical } \\
\text { parameters }\end{array}$} & \multicolumn{4}{|l|}{ Stage I } & \multicolumn{4}{|l|}{ Stage II } \\
\hline & No RT & $\begin{array}{l}\text { Neoadjuvant } \\
\text { RT }\end{array}$ & $\begin{array}{l}\text { adjuvant } \\
\mathrm{RT}\end{array}$ & $P$ & No RT & $\begin{array}{l}\text { Neoadjuvant } \\
\text { RT }\end{array}$ & $\begin{array}{l}\text { adjuvant } \\
\text { RT }\end{array}$ & $P$ \\
\hline Age & & & & 0.626 & & & & 0.001 \\
\hline$<60$ & $471(29.2)$ & $26(29.5)$ & $123(31.7)$ & & $1822(22.4)$ & 193(35.5) & 1297(30.9) & \\
\hline$\geq 60$ & $1142(70.8)$ & $62(70.5)$ & $265(68.3)$ & & $6322(77.6)$ & $351(64.5)$ & 2903(69.1) & \\
\hline $\begin{array}{l}\text { Year of } \\
\text { diagnosis }\end{array}$ & & & & 0.001 & & & & 0.001 \\
\hline $2004-2009$ & 737(45.7) & $34(38.6)$ & $235(60.6)$ & & $3226(39.6)$ & $158(29.0)$ & 2101(50.0) & \\
\hline 2010-2015 & $876(54.3)$ & $54(61.4)$ & 153(39.4) & & $4918(60.4)$ & $386(71.0)$ & 2099(50.0) & \\
\hline Sex & & & & 0.002 & & & & 0.133 \\
\hline Female & $916(56.8)$ & $44(50.0)$ & $183(47.2)$ & & $4038(49.6)$ & $278(51.1)$ & 2012(47.9) & \\
\hline Male & 697(43.2) & $44(50.0)$ & $205(52.8)$ & & $4106(50.4)$ & $266(48.9)$ & $2188(52.1)$ & \\
\hline Race & & & & 0.012 & & & & 0.009 \\
\hline Black & $161(10.0)$ & 12(13.6) & $58(14.9)$ & & $751(9.2)$ & $59(10.8)$ & $394(9.4)$ & \\
\hline White & 1282(79.5) & 72(81.8) & $300(77.3)$ & & $6773(83.2)$ & $466(85.7)$ & $3492(83.1)$ & \\
\hline Other & $170(10.5)$ & $4(4.5)$ & $30(7.7)$ & & $620(7.6)$ & 19(3.5) & $314(7.5)$ & \\
\hline $\begin{array}{l}\text { Primary tumor } \\
\text { Site }\end{array}$ & & & & 0.001 & & & & 0.001 \\
\hline Head & $880(54.6)$ & 65(73.9) & $251(64.7)$ & & $6285(77.2)$ & $461(84.7)$ & $3314(78.9)$ & \\
\hline Body & 233(14.4) & $11(12.5)$ & $56(14.4)$ & & $512(6.3)$ & $36(6.6)$ & $270(6.4)$ & \\
\hline tail & $381(23.6)$ & $6(6.8)$ & $54(13.9)$ & & $904(11.1)$ & $22(4.0)$ & $398(9.5)$ & \\
\hline Pancreatic duct & $26(1.6)$ & $1(1.1)$ & $7(1.8)$ & & $100(1.2)$ & $1(0.2)$ & $44(1.0)$ & \\
\hline Overlapping & $93(5.8)$ & $5(5.7)$ & $20(5.2)$ & & $343(4.2)$ & $24(4.4)$ & $174(4.1)$ & \\
\hline $\begin{array}{l}\text { Pathological } \\
\text { grade }\end{array}$ & & & & 0.001 & & & & 0.001 \\
\hline 1 & $315(19.5)$ & $12(13.6)$ & $57(14.7)$ & & $768(9.4)$ & $42(7.7)$ & $370(8.8)$ & \\
\hline II & $608(37.7)$ & $22(25.0)$ & $202(52.1)$ & & $3781(46.4)$ & $166(30.5)$ & 2094(49.9) & \\
\hline III & $272(16.9)$ & $18(20.5)$ & $89(22.9)$ & & $2878(35.3)$ & $106(19.5)$ & $1450(34.5)$ & \\
\hline IV & $29(1.8)$ & $0(0)$ & $5(1.3)$ & & $112(1.4)$ & $8(1.5)$ & $53(1.3)$ & \\
\hline Unknown & $389(24.1)$ & $36(40.9)$ & $35(9.0)$ & & $605(7.4)$ & $222(40.8)$ & $233(5.5)$ & \\
\hline $\mathrm{T}$ & & & & 0.001 & & & & 0.002 \\
\hline 1 & $636(39.4)$ & $20(22.7)$ & $115(29.6)$ & & 157(1.9) & $2(0.4)$ & $75(1.8)$ & \\
\hline 2 & $977(60.6)$ & 68(77.3) & $273(70.4)$ & & $582(7.1)$ & $23(4.2)$ & $325(7.7)$ & \\
\hline
\end{tabular}




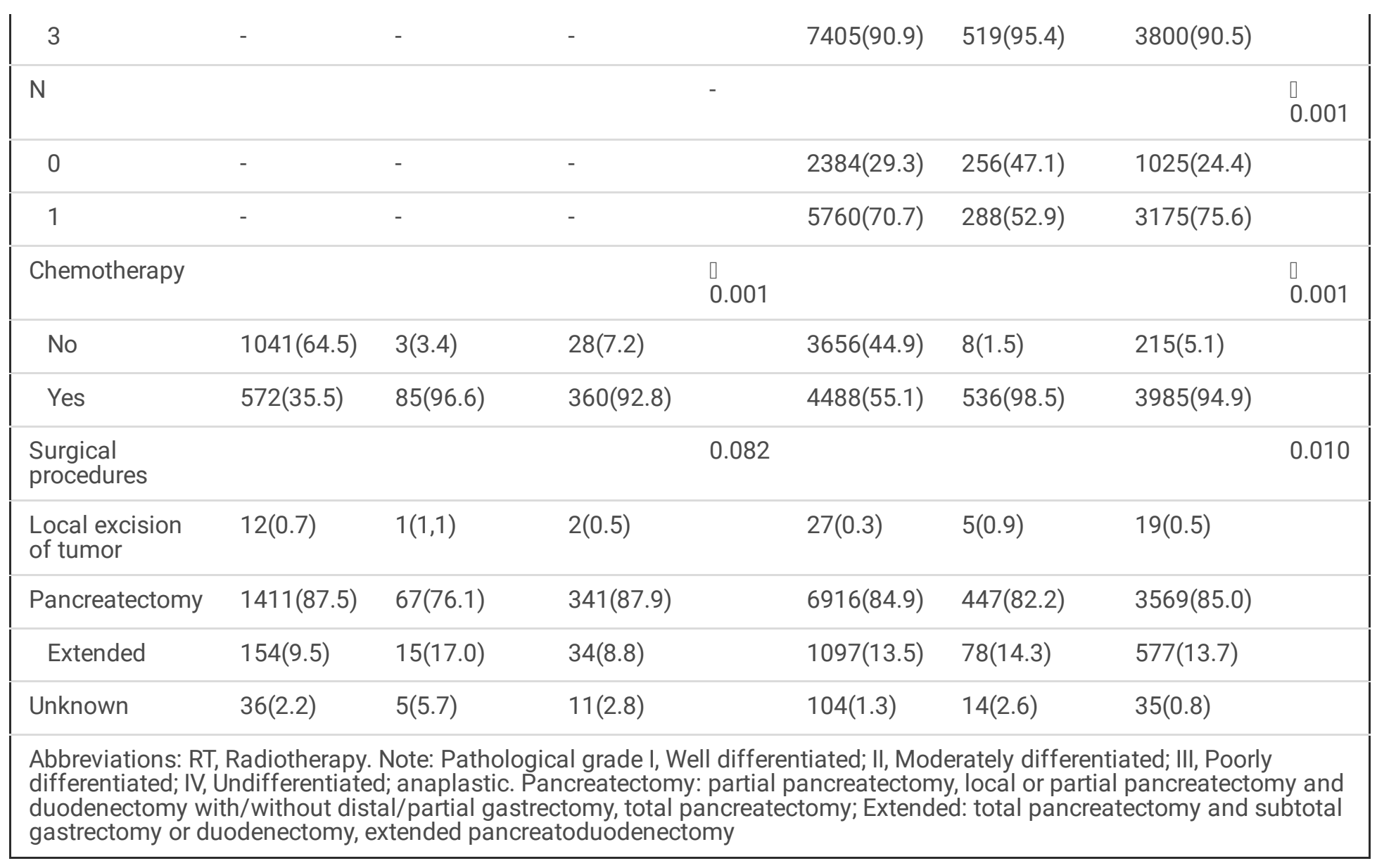

Table2 Univariate analysis of clinical features affecting the prognosis of pancreatic cancer patients 


\begin{tabular}{|c|c|c|c|c|c|c|}
\hline \multirow[t]{2}{*}{ Clinical parameters } & \multicolumn{3}{|c|}{ Stage I } & \multicolumn{3}{|c|}{ Stage II } \\
\hline & $\mathrm{HR}$ & $95 \% \mathrm{Cl}$ & $P$ & $\mathrm{HR}$ & $95 \% \mathrm{Cl}$ & $\mathrm{P}$ \\
\hline \multicolumn{7}{|l|}{ Age } \\
\hline$<60$ & Ref & & & Ref & & \\
\hline$\geq 60$ & 2.063 & $1.772-2.401$ & $\llbracket 0.001$ & 1.263 & $1.204-1.324$ & $\varangle 0.001$ \\
\hline \multicolumn{7}{|l|}{ Year of diagnosis } \\
\hline 2004-2009 & Ref & & & Ref & & \\
\hline 2010-2015 & 0.897 & $0.785-1.024$ & 0.109 & 0.866 & $0.830-0.902$ & $\varangle 0.001$ \\
\hline \multicolumn{7}{|l|}{ Sex } \\
\hline Female & Ref & & & Ref & & \\
\hline Male & 1.279 & $1.131-1.446$ & $\llbracket 0.001$ & 1.042 & $1.000-1.085$ & 0.049 \\
\hline \multicolumn{7}{|l|}{ Race } \\
\hline Black & Ref & & & Ref & & \\
\hline White & 1.077 & $0.880-1.319$ & 0.470 & 0.959 & $0.895-1.028$ & 0.242 \\
\hline Other & 0.760 & $0.567-1.020$ & 0.067 & 0.911 & $0.823-1.008$ & 0.072 \\
\hline \multicolumn{7}{|l|}{ Primary tumor Site } \\
\hline Head & Ref & & & Ref & & \\
\hline Body & 0.831 & $0.690-1.002$ & 0.052 & 0.975 & $0.894-1.063$ & 0.568 \\
\hline tail & 0.606 & $0.512-0.718$ & $\bowtie 0.001$ & 0.909 & $0.848-0.974$ & 0.007 \\
\hline Pancreatic duct & 0.856 & $0.536-1.368$ & 0.517 & 0.876 & $0.725-1.058$ & 0.169 \\
\hline Overlapping & 0.773 & $0.583-1.025$ & 0.074 & 1.072 & $0.968-1.188$ & 0.179 \\
\hline \multicolumn{7}{|l|}{ Pathological grade } \\
\hline 1 & Ref & & & Ref & & \\
\hline II & 1.811 & $1.499-2.187$ & $\llbracket 0.001$ & 1.440 & $1.331-1.559$ & $\nabla 0.001$ \\
\hline III & 2.570 & $2.085-3.168$ & $\varangle 0.001$ & 1.902 & $1.754-2.061$ & $\varangle 0.001$ \\
\hline IV & 1.746 & $1.056-2.889$ & 0.030 & 1.684 & $1.394-2.033$ & $\otimes 0.001$ \\
\hline Unknown & 1.039 & $0.829-1.303$ & 0.739 & 1.147 & $1.031-1.276$ & 0.012 \\
\hline \multicolumn{7}{|l|}{$\mathrm{T}$} \\
\hline 1 & Ref & & & Ref & & \\
\hline 2 & 1.371 & $1.201-1.564$ & $\varangle 0.001$ & 1.315 & $1.113-1.554$ & 0.001 \\
\hline 3 & - & & & 1.295 & $1.113-1.506$ & 0.001 \\
\hline \multicolumn{7}{|l|}{$\mathrm{N}$} \\
\hline 0 & - & & & Ref & & \\
\hline 1 & - & & & 1.446 & $1.380-1.516$ & $\varangle 0.001$ \\
\hline Chemotherapy & & & & & & \\
\hline
\end{tabular}




\begin{tabular}{|c|c|c|c|c|c|c|}
\hline No & Ref & & & Ref & & \\
\hline Yes & 1.056 & 0.933-1.194 & 0.389 & 0.637 & $0.610-0.666$ & $\otimes 0.001$ \\
\hline \multicolumn{7}{|l|}{ Surgical procedures } \\
\hline Local excision of tumor & Ref & & & Ref & & \\
\hline Pancreatectomy & 0.900 & $0.428-1.894$ & 0.782 & 0.752 & $0.551-1.026$ & 0.072 \\
\hline Extended & 0.962 & $0.447-2.071$ & 0.922 & 0.820 & $0.598-1.123$ & 0.215 \\
\hline Unknown & 1.212 & $0.529-2.775$ & 0.650 & 0.791 & $0.550-1.135$ & 0.203 \\
\hline \multicolumn{7}{|l|}{ Radiotherapy } \\
\hline No & & Ref & & Ref & & \\
\hline neoadjuvant RT & 1.468 & $1.099-1.960$ & 0.009 & 0.690 & $0.617-0.770$ & $\unrhd 0.001$ \\
\hline adjuvant RT & 1.193 & $1.028-1.384$ & 0.020 & 0.790 & $0.756-0.825$ & $\unrhd 0.001$ \\
\hline
\end{tabular}

Table3 Multivariate analysis of clinical features affecting the prognosis of pancreatic cancer patients 


\begin{tabular}{|c|c|c|c|c|c|c|}
\hline \multirow[t]{2}{*}{ Clinical parameters } & \multicolumn{3}{|c|}{ Stage I } & \multicolumn{3}{|c|}{ Stage II } \\
\hline & $\mathrm{HR}$ & $95 \% \mathrm{Cl}$ & $P$ & $\mathrm{HR}$ & $95 \% \mathrm{Cl}$ & $\mathrm{P}$ \\
\hline \multicolumn{7}{|l|}{ Age } \\
\hline$<60$ & Ref & & & Ref & & \\
\hline$\geq 60$ & 1.894 & $1.621-2.213$ & $\varangle 0.001$ & 1.220 & $1.162-1.280$ & $\varangle 0.001$ \\
\hline \multicolumn{7}{|l|}{ Year of diagnosis } \\
\hline 2004-2009 & Ref & & & Ref & & \\
\hline 2010-2015 & 0.943 & $0.823-1.080$ & 0.394 & 0.887 & $0.850-0.926$ & $\varangle 0.001$ \\
\hline \multicolumn{7}{|l|}{ Sex } \\
\hline Female & Ref & & & Ref & & \\
\hline Male & 1.172 & $1.035-1.327$ & 0.012 & 1.055 & $1.013-1.100$ & 0.010 \\
\hline \multicolumn{7}{|l|}{ Race } \\
\hline Black & Ref & & & Ref & & \\
\hline White & 0.971 & $0.790-1.192$ & 0.776 & 0.907 & $0.846-0.973$ & 0.006 \\
\hline Other & 0.705 & $0.524-0.949$ & 0.021 & 0.889 & $0.802-0.985$ & 0.024 \\
\hline \multicolumn{7}{|l|}{ Primary tumor Site } \\
\hline Head & Ref & & & Ref & & \\
\hline Body & 0.820 & $0.679-0.989$ & 0.038 & 1.049 & $0.961-1.145$ & 0.281 \\
\hline tail & 0.698 & $0.587-0.830$ & $₫ 0.001$ & 0.953 & $0.888-1.023$ & 0.181 \\
\hline Pancreatic duct & 1.150 & $0.717-1.845$ & 0.563 & 0.789 & $0.653-0.954$ & 0.014 \\
\hline Overlapping & 0.772 & $0.581-1.027$ & 0.076 & 1.088 & $0.982-1.205$ & 0.107 \\
\hline \multicolumn{7}{|l|}{ Pathological grade } \\
\hline I & Ref & & & Ref & & \\
\hline II & 1.719 & $1.418-2.084$ & $\otimes 0.001$ & 1.466 & $1.354-1.587$ & $\varangle 0.001$ \\
\hline III & 2.318 & $1.867-2.878$ & $\otimes 0.001$ & 1.947 & $1.796-2.112$ & $\varangle 0.001$ \\
\hline IV & 1.861 & $1.121-3.089$ & 0.016 & 1.807 & $1.495-2.183$ & $\otimes 0.001$ \\
\hline Unknown & 1.010 & $0.803-1.268$ & 0.935 & 1.273 & $1.142-1.419$ & $\varangle 0.001$ \\
\hline \multicolumn{7}{|l|}{$\mathrm{T}$} \\
\hline 1 & Ref & & & Ref & & \\
\hline 2 & 1.412 & $1.234-1.615$ & 0.000 & 1.274 & $1.078-1.506$ & 0.004 \\
\hline 3 & - & & & 1.450 & $1.245-1.690$ & $\otimes 0.001$ \\
\hline \multicolumn{7}{|l|}{$N$} \\
\hline 0 & - & & & Ref & & \\
\hline 1 & - & & & 1.543 & $1.469-1.621$ & $\varangle 0.001$ \\
\hline Chemotherapy & & & & & & \\
\hline
\end{tabular}




\begin{tabular}{|c|c|c|c|c|c|c|}
\hline No & Ref & & & Ref & & \\
\hline Yes & 0.827 & $0.727-0.940$ & 0.004 & 0.628 & $0.598-0.660$ & $\nabla 0.001$ \\
\hline \multicolumn{7}{|l|}{ Surgical procedures } \\
\hline Local excision of tumor & \multicolumn{3}{|l|}{ Ref } & \multicolumn{3}{|l|}{ Ref } \\
\hline Pancreatectomy & 0.677 & $0.320-1.433$ & 0.308 & 0.647 & $0.474-0.884$ & 0.006 \\
\hline Extended & 0.642 & $0.296-1.393$ & 0.263 & 0.702 & $0.512-0.963$ & 0.028 \\
\hline Unknown & 1.208 & $0.524-2.784$ & 0.657 & 0.713 & $0.496-1.025$ & 0.068 \\
\hline \multicolumn{7}{|l|}{ Radiotherapy } \\
\hline No & \multicolumn{3}{|l|}{ Ref } & \multicolumn{3}{|l|}{ Ref } \\
\hline neoadjuvant RT & 1.434 & $1.051-1.957$ & 0.023 & 0.975 & $0.868-1.095$ & 0.668 \\
\hline adjuvant RT & 1.095 & 0.918-1.307 & 0.314 & 0.904 & $0.861-0.950$ & $\unrhd 0.001$ \\
\hline \multicolumn{7}{|c|}{$\begin{array}{l}\text { Abbreviations: RT, Radiotherapy. Note: Pathological grade I, Well differentiated; II, Moderately differentiated; III, Poorly } \\
\text { differentiated; IV, Undifferentiated; anaplastic. Pancreatectomy: partial pancreatectomy, local or partial pancreatectomy anc } \\
\text { duodenectomy with/without distal/partial gastrectomy, total pancreatectomy; Extended: total pancreatectomy and subtotal } \\
\text { gastrectomy or duodenectomy, extended pancreatoduodenectomy }\end{array}$} \\
\hline
\end{tabular}

\section{Figures}


Searching SEER database for case of primary pancreatic cancer patients

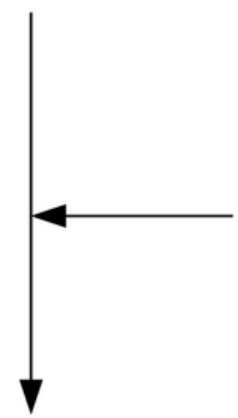

Searching criteria:

1) year of diagnosis:2004-2015

2) age $\geq 18$

3) diagnosed as stage I/II pancreatic cancer(AJCC staging manual)

4) surgical treatment received

16456 patients were identified

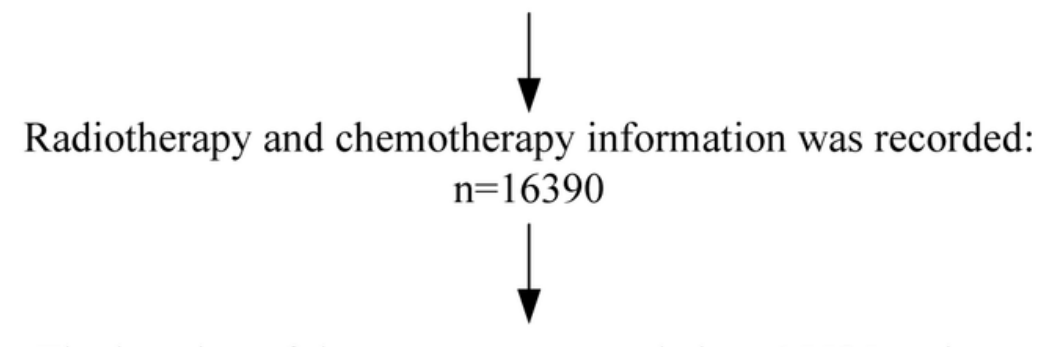

The location of the tumor was recorded: $\mathrm{n}=15525$ patients

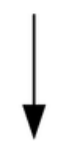

Survival time was at least one month:

14977 patients included in the study

Fig.1

Figure 1

Flow chart showed selection of PC patients registered in SEER from 2004 to 2015 in this study
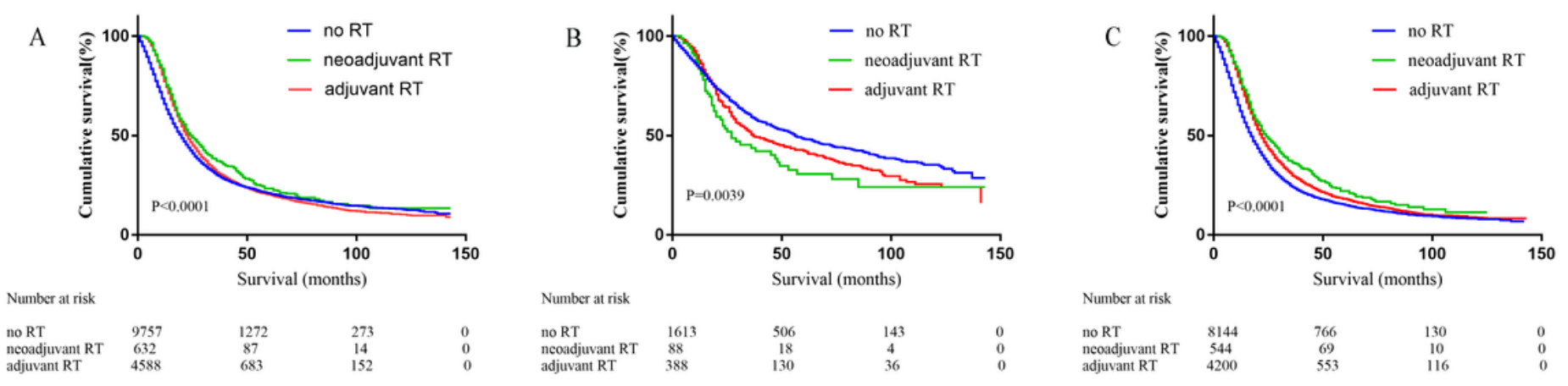

Fig. 2

Figure 2 
Overall survival of patients stratified by RT (A) Overall survival of 14977 cases of stage I/Il patients treated without RT ( $n=9757$ ) versus patients treated with neoadjuvant $R T(n=632)$ versus patients treated with adjuvant $R T(n=4588)(P<0.0001)$. (B) Plot of overall survival for 2089 stage I patients stratified by RT use. Patients not received RT ( $n=1613)$ versus patients received neoadjuvant RT ( $n=88)$ versus patients treated with adjuvant RT $(n=388)(P=0.0039)$. (C) Plot of overall survival for 12888 stage II patients stratified by RT use. Patients not received RT ( $n=8144)$ versus patients received neoadjuvant RT ( $n=544)$ versus patients treated with adjuvant RT $(n=4200)(P<0.0001)$.
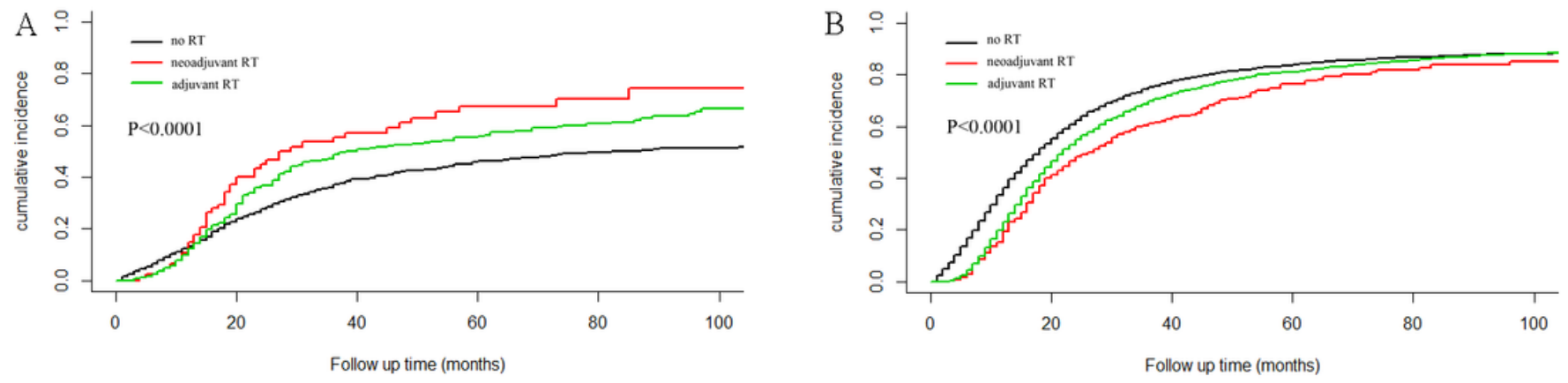

Fig. 3

\section{Figure 3}

Competitive risk analysis for cancer-related death of patients stratified by RT (A) Cancer-related death of stage I patients caused by RT use or not. $(P<0.0001)$ (B) Cancer-related death of patients stage Il patients caused by RT. $(P<0.0001)$ 